\title{
La representación de la mujer trabajadora en los espacios radiofónicos. Una nueva realidad en las ondas.
}

\section{The representation of working women on radio. A new fact on this mass media.}

\author{
Jose Manuel Frasquier Marchal \\ imfrasquier@hotmail.com \\ UNED. Universidad Nacional de Educación a Distancia
}

\section{Resumen}

En nuestra Sociedad de la Información los medios de comunicación se convierten en constructores y transmisores del hecho cultural al actuar como mediadores y agentes fundamentales en la concepción de nuestro entorno. En el caso de la radio, nos encontramos con una forma de comunicar muy cercana, que actúa como referente ante las nuevas circunstancias sociales. En particular, de las circunstancias sociales que acontecen con la incorporación de la mujer a parcelas profesionales de las que antes había quedado excluida, como son las carreras judicial y fiscal. Por todo ello, el presente trabajo plantea como objetivo exponer, a través de un análisis del discurso de orden cualitativo e inductivo, cómo en los espacios de audiencias generalistas y de programación convencional, se nombra a estas mujeres y bajo qué patrones lingüísticos se construyen estas nuevas realidades, que quedan representadas en los espacios radiofónicos españoles de ámbito nacional: COPE, RNE, SER y Onda Cero.

\section{Palabras clave}

Radio; Análisis del discurso; Género; Mujeres trabajadoras; Carrera fiscal; Fiscala; Carrera judicial; Jueza.

\section{Abstract}

In our Information Society the mass media become as builders and transmitters of our culture acting as mediators and main agents in the conception of our current 
context. In this way, we come across the radio, a way of communicating that sets linguistic guides. Also in the face of new social circumstances such as the incorporation of women into professional fields in judicial career or fiscal career from that had previously been excluded. Therefore, we want to expose here how the radio talks about these women and what linguistic patterns are used to build the new realities that are represented on the Spanish radio programs through an analysis of the discourse of qualitative and inductive order of networks: COPE, RNE, SER and Onda Cero.

\section{Keywords}

Radio; Discourse analysis; Gender; Working women; Fiscal career; Female fiscal; Judicial career; female judge.

Recibido:29/05/2018

Aceptado:23/06/2018

DOI: http://dx.doi.org/10.5557/IIMEI9-N16-001025

Descripción propuesta: Frasquier Marchal, Jose Manuel, 2018. La representación de la mujer trabajadora en los espacios radiofónicos. Una nueva realidad en las ondas. Métodos de Información, 9(16), 1-25

\section{Introducción}

En nuestra Sociedad de la Información los medios de comunicación, que de por sí son medios sociales (Bruns 2015), actúan como mediadores llegando a convertirse en herramientas condicionadoras y determinantes, al contextualizarse como agentes fundamentales en la concepción de nuestro entorno, a veces con carácter de exclusividad. Su influencia es tal, que se han convertido en imprescindibles (Esteve, 2003), en muchos casos actuando como catalizadores para la transformación social (Rimal, Chung y Dhungana 2015), llegando incluso a convertirse en constructores de la realidad (Castells, 2007). Actualmente, en la era de internet de banda ancha, existen muchas más oportunidades que posibilitan su uso selectivo, contrariamente a lo que acontecía anteriormente con los medios tradicionales (Hendriks Vettehen et al. 2017). Unas circunstancias sobre las que no hay que descartar el interés en la difusión de unos discursos públicos cuyo objetivo es el avance, el sostenimiento y la legitimación del orden neoliberal (Krzyżanowski 2016). 
"Media are among the principal social agents in many societies around the world. Television, magazines, newspapers, radio, cinema, advertising, the Internet, and other so-called "new media" or "new technologies" occupy— if not invade-much of our leisure time, and indeed our working time" (LópezGuimera et al. 2010, p. 388).

Entre estos medios se encuentra la radio, una forma de comunicación que impregna nuestra vida diaria de forma sutil, formando parte de nuestra fonosfera (Alonso González 2004). La radio ha sobrevivido al nuevo panorama mediático instalado en el ciberespacio modificando, tanto el sistema de producción de sus contenidos como su transmisión y consumo, con la aparición de la radio on-line y las bitcasters (Ribes 2001), que permiten navegar en un entorno virtual, facilitando su audición bajo demanda, mediante los formatos podcast y las diferentes aplicaciones que las emisoras utilizan (Bonet 2017). Todo ello conlleva la aparición de nuevos usos, que llegan a romper con el paradigma espacio-temporal de la radio off-line, convirtiendo la nueva escucha en una acción programada, asíncrona y no estacionaria (Gallego Pérez 2010).

Estas innovaciones tecnológicas, sin embargo, no han conseguido eliminar una propiedad consustancial de la radio, inherente a este medio desde su origen: el vínculo tan estrecho que establece con quienes la sintonizan, por ser un modelo instantáneo, cercano, próximo y flexible de comunicación (Fernández Sande y Peinado Miguel 2012), que se sirve exclusivamente de códigos sonoros, entre los que se encuentra la voz, para realizar una transmisión imago-acústica (Lozano, 2006).

La voz humana es una herramienta cargada de un inmensurable poder emotivo, con una gran capacidad para la persuasión, si se articula de forma conveniente, y con la que se pronuncian las palabras que actúan como elementos esenciales del lenguaje al que pertenecen. Un lenguaje que moldea de forma invisible la forma de pensar de las personas y sus acciones (Tannen 1996), convirtiéndose en transmisor de pensamientos e ideas y no sólo de 
comunicación (Ibáñez 2003), pudiendo llegar a ser una herramienta de acción, en cuanto que es posible hacer cosas con él (Austin, 1991). Esto es debido a que las palabras no son neutras sino que adquieren unos significados concretos, que son los valores que les asignan quienes las utilizan, según en qué contextos se crean, articulan y difunden.

\subsection{Lenguaje mediático y sexismo}

Con las palabras y con el lenguaje describimos realidades que, cuando se da la circunstancia de hacerlo desde una perspectiva discriminatoria y de desigualdad para referenciar al género, las convertimos en palabras y lenguaje sexistas. Al utilizar estas herramientas discursivas así, se construyen representaciones valorizadas, diferenciadoras y excluyentes de lo masculino con respecto a lo femenino.

Esta coyuntura acontece debido a los modelos de creencias subyacentes de quienes utilizan el discurso, no de él mismo (Van Dijk 2003), ya que el lenguaje no es misógino en su estructura, sino en su uso (Wittig 2005). Así sucede con el castellano, que no es sexista per se sino que lo convertimos en sexista al utilizarlo (García Messeguer 2001). De esta manera, los discursos sexistas, como ocurre con los homofóbicos y los racistas, están basados en prejuicios heredados que se transfieren de generación en generación y se almacenan en forma de modelos mentales y del conocimiento (Wodak 2006).

También cuando la radio difunde unos contenidos que han sido producidos y re-producidos según unos patrones asimétricos por razón de género, los convierte en contenidos sexistas. Es decir, la radiodifusión consiste en utilizar el discurso oral, potencialmente modélico para las audiencias, como instrumento de discrimación, aunque connaturalmente no lo sea.

La forma en la que los medios transmiten una representación concreta de la realidad los convierte en impulsores de la creación de estereotipos con los que se llega a construir la diferencia y la alteridad (First 2016). Así acontece, por ejemplo, cuando una voz se construye mediante modulaciones tímbricas diferenciadas para varones y mujeres, porque han sido referenciadas en 
patrones y etiquetas sociales y culturales, por lo que sirven para mantener y reforzar los estereotipos de género (Fasoli, Mazzurega y Sulpizio 2017).

Esto se debe a que los estereotipos que más se reproducen en los hábitos sociales son aquellos que han sido utilizados en mayor medida con el paso del tiempo, es decir, los que son altamente comunicables en las conversaciones (Biernat et al. 2018), en cuya difusión masiva influyen los propios medios, como el radiofónico.

\subsection{Objetivo}

Ante lo expuesto nos hemos preguntado: ¿Cómo es el discurso que el medio radiofónico difunde, analizado desde una perspectiva de género?

Nuestro objetivo es mostrar el modo en el que un medio de comunicación tan consumido y tan influyente como es el radiofónico ${ }^{1}$ refleja la nueva realidad que acontece. Y cómo lo hace, acerca de aquellas mujeres que han decidido abandonar el espacio privado en el que se las ubicó primitivamente, para saltar a otro espacio, profesional, público y de reconocido prestigio. En concreto, en el espacio profesional de la carrera judicial y la carrera fiscal, es decir, en el caso de mujeres juezas y fiscalas.

Una novedosa coyuntura de la que sabemos que, como tal, necesita una nueva denominación para referirla porque hasta ahora, al ser un espacio privativo de los varones, se utilizaba el género gramatical masculino para hacerlo. Así, queremos analizar si en la actualidad el lenguaje radiofónico se ha adaptado a estas nuevas circunstancias y cómo menciona a estas mujeres, ya que sabemos que no existe forma de referirse lingüísticamente a lo que no es, y por ello lo novedoso necesita de un lenguaje para referenciarlo.

\subsection{La mujer profesional en los diccionarios}

Como paso previo a la descripción del método utilizado para elaborar este análisis vamos a exponer cómo se manifiestan las instituciones encargadas de velar por el correcto uso del español al respecto de las palabras con las cuales nombrar a las juezas y a las fiscalas. 
En nuestra búsqueda sobre el término fiscala encontramos que este vocablo está aceptado y que consecuentemente debe utilizarse en sus diferentes acepciones, tanto en el Diccionario de la lengua española de la Real Academia de la Lengua Española (RAE 2014, p. 1033), como el Diccionario esencial (RAE 2006, p. 678), la Nueva Gramática de la Lengua Española (RAE 2010, p. 28), el Diccionario panhispánico de dudas (RAE, 2005), así como en el Diccionario de uso del español de María Moliner (Moliner 2007, p. 1367).

En los mismos diccionarios, también está admitido el uso de palabra jueza, es decir, el Diccionario de la Real Academia de la Lengua Española (RAE 2014, p. 1291), el Diccionario esencial (RAE 2006, p. 859), la Nueva Gramática de la Lengua Española (RAE 2010, p. 28), el Diccionario panbispánico de dudas (RAE, 2005), y el de uso del español de María Moliner (Moliner 2007, p. 1713).

\section{Material y métodos}

En este trabajo realizamos un análisis del discurso de orden cualitativo e inductivo, al ser su objeto de estudio de naturaleza discursiva (Santander, 2011). Además, es naturalista ya que no se actúa sobre los discursos seleccionados (Taylor y Bodgan 1987), interpretativo (Valles 2000; Hernández, Fernández y Baptista 2006), y está centrado en la importancia de la estructura, significación y empleo del lenguaje (Feltham-King y Macleod 2016).

Considerando que el discurso es un arma constructiva fundamental que, además de contribuir a la creación de una realidad, adquiere un papel relevante como instrumento ideológico y de poder (Fernández Martínez 2007), hemos adoptado una posición de estudio cimentada en el cuestionamiento crítico que identifique los usos lingüísticos, las expresiones y los vocablos que se utilizan para, desvelando lo oculto, ofrecer visibilidad sobre un mundo de representaciones (Hernández, Fernández y Baptista 2006). De este modo, reconociendo el gran poder de influencia de la radio nos hemos alejado de toda candidez o ingenuidad que la considera como un medio banal de comunicación o como una simple forma de producción y difusión de entretenimiento.

Todas estas consideraciones hacen que este estudio tome el enfoque del Análisis Crítico del Discurso (ACD). 
Así, adquiriremos una posición crítica entendiendo la capacidad que poseen los discursos no solo para describir la práctica social sino también para definirla y darle forma, ya que el poder también se puede ejercer usando los discursos, al inducir o suprimir el comportamiento (Burke 2015), con el objetivo de desvelar lo que disfrazan, encierran o esconden las alocuciones radiofónicas en las que se hace referencia a las mujeres que trabajan en la carrera judicial. Dado que los signos lingüísticos y semánticos no son neutros, han de ser estudiados tanto desde una perspectiva denotativa como también desde una perspectiva connotativa. Todo este ejercicio se ejecutará estimando el componente de inferencia que posee toda comunicación humana, que va más allá de tratarla como un proceso simplista de codificación y decodificación de contenidos (Sperber y Wilson 2004). Consiste en una estrategia metodológica que considera que el análisis de la noticia requiere además de un nivel textual, un nivel contextual, es decir, que sean considerados otros aspectos como los de su representación (Van Dijk 1990).

Nuestro itinerario analítico de trabajo nos lleva a adoptar el modelo de análisis de Foucault (2002) considerando que conceptualiza el discurso como sustitución de ideología. Este autor deconstruyó la percepción de las relaciones de poder unidireccionales entre las instituciones y la ciudadanía al entender que se distribuyen como si fueran vasos capilares (Matarese y Van Nijnatten 2015), y desarrolló la idea de que quienes se encuentran en el poder controlan la retórica que se emplea y subordinan la retórica de las minorías que no están bajo control político (Voss y Van Dyke 2001).

\subsection{La población y la muestra}

El análisis ejecutado en este trabajo se ha elaborado sobre una población compuesta por los espacios radiofónicos que ha sido seleccionada de acuerdo a tres parámetros:

1.- Según la tipología de programación. Los espacios pertenecen al formato de radio convencional también denominado como radio total o tradicional (Martí, 2004), poseen un target generalista y mayoritario, y sus contenidos se han transmitido en primer lugar en formato off-line, y posteriormente on-line. 
2.- Según la cobertura geográfica han sido elegidos los espacios que se difunden en territorio español.

3.- Según la temporalidad de las emisiones han tenido que ser emitidos durante la temporada mediática 2014/2015.

Así, nos encontramos con los programas pertenecientes a las parrillas de las cuatro empresas de radiodifusión sonora con mayor cantidad de audiencia dentro del territorio español ${ }^{2}$ según se detalla en la Tabla 1.

\begin{tabular}{|c|c|c|c|}
\hline \multirow{4}{*}{$\begin{array}{l}\text { CRITERIOS } \\
\text { PARA LA } \\
\text { SELECCIÓN } \\
\text { DE LA } \\
\text { POBLACIÓN }\end{array}$} & $\begin{array}{l}\text { 1.- TIPOLOGÍA DE } \\
\text { PROGRAMACIÓN: }\end{array}$ & Convencional & \multirow{4}{*}{$\begin{array}{l}\text {-COPE (Cadena de } \\
\text { Ondas Populares } \\
\text { Españolas). } \\
\text {-ONDA CERO } \\
\text { RADIO. } \\
\text {-RADIO 1. RNE } \\
\text { (Radio Nacional de } \\
\text { España). } \\
\text {-SER (Sociedad } \\
\text { Española de } \\
\text { Radiodifusión). }\end{array}$} \\
\hline & \multirow[t]{2}{*}{$\begin{array}{l}\text { 2.- COBERTURA } \\
\text { GEOGRÁFICA: }\end{array}$} & $\begin{array}{l}\text { En cadena. } \\
\text { Emisión } \\
\text { off-line en todo } \\
\text { el territorio } \\
\text { español. }\end{array}$ & \\
\hline & & $\begin{array}{l}\text { Emisión on-line } \\
\text { desde el } \\
\text { territorio } \\
\text { español. }\end{array}$ & \\
\hline & 3.-TEMPORALIDAD: & $\begin{array}{l}\text { Temporada } \\
\text { mediática } \\
2014 / 2015 .\end{array}$ & \\
\hline
\end{tabular}

Tabla 1. Criterios para la selección de la población. (Fuente: elaboración propia).

En cuanto a la muestra se refiere, ésta se ha seleccionado según la técnica de muestreo por agrupamiento o conglomerados monoetápicos entendiendo que cada uno de estos es cada día de emisión. Así, durante el transcurso de cada uno se encuentran ordenados de forma coherente los contenidos que se difunden (Faus 1981), funcionando como unidades independientes (Tenorio 2012), dentro del conjunto de la parrilla que es la seña de identidad de una radio (Perona, 2012). Además, es monoetápico porque se miden todos los elementos que forman parte de cada conglomerado, es decir, todos los programas que se difunden a lo largo del mismo día en sus 24 horas de emisión, sin ejecutar submuestreo sobre ellos.

Para la selección de los conglomerados, o días de emisión, se han seguido dos procedimientos: 
1. Por muestreo probabilístico aleatorio simple con réplicas (Vivanco 2006), que es equiprobable (Alaminos et. al 2006). Según éste ha sido seleccionada la semana de programación que transcurre desde el lunes 15 al domingo 21 de diciembre de 2014.

2. Por muestreo estratificado. Así, se han añadido más conglomerados para conseguir información adicional sobre variables de estratificación con la que se pretende mejorar la selección de la población y la precisión del estudio (Vivanco, 2005). Éstos son aquellos días en los que se han radiado contenidos informativos sobre acontecimientos que tienen una relación directa con el género, el objetivo de este estudio. Consiste en el estudio de casos que interesa realizar de una selección orientada por la información según la cual dicha muestra está condicionada y determinada por la clase de investigación en la que se incluye (Flyvbjerg 2006). En nuestro caso específico se han elegido siete días en los que se producen informaciones específicas por lo que se trata de un muestreo de aquellos casos que exponen con intensidad aquello que se investiga (Patton 2015).

Los conglomerados seleccionados, o días de emisión, son los que se detallan en la Tabla 2.

\begin{tabular}{|c|l|l|}
\hline \multirow{2}{*}{$\begin{array}{c}\text { MUESTRA } \\
\text { seleccionada } \\
\text { por: }\end{array}$} & $\begin{array}{l}\text { 1.- SELECCIón } \\
\text { PROBABILÍSTICA } \\
\text { ALEATORIA: }\end{array}$ & $\begin{array}{l}\text { Parrilla semanal de } \\
\text { programación desde el } \\
\text { lunes 15 al domingo 21 } \\
\text { de Diciembre de 2014. }\end{array}$ \\
\cline { 2 - 3 } $\begin{array}{c}\text { conglomerados } \\
\text { monoetápicos }\end{array}$ & $\begin{array}{l}\text { 2.- SELECCIÓN } \\
\text { ESTRATIFICADA. }\end{array}$ & $\begin{array}{l}\text { Jueves 8 de Enero. } \\
\text { Selección intencionada de } \\
\text { conglomerados del año 2015: } \\
\text { Martes 17 de Febrero. } \\
\text { Martes 14 de Abril. } \\
\text { Domingo 8 de Marzo. } \\
\text { Sábado 4 de Julio. } \\
\text { Domingo 5 de Julio. }\end{array}$ \\
\hline
\end{tabular}

Tabla 2. Procedimiento metodológico para la selección de la muestra. (Fuente: elaboración propia).

Por su parte, los espacios que son analizados son los que se detallan en la Tabla 3. 


\begin{tabular}{|c|c|}
\hline $\begin{array}{l}\text { POBLACIÓN } \\
\text { Cadenas y plataformas que difunden } \\
\text { espacios radiofónicos. }\end{array}$ & $\begin{array}{l}\quad \text { MUESTRA } \\
\text { Programas y espacios de difusión } \\
\text { nacional. }\end{array}$ \\
\hline $\begin{array}{l}\text { COPE (Cadena de Ondas Populares } \\
\text { Españolas) }\end{array}$ & $\begin{array}{l}\text { La mañana. } \\
\text { El espejo. } \\
\text { La tarde. } \\
\text { La linterna. } \\
\text { El partido de las doce. } \\
\text { Fin de semana. } \\
\text { Mediodía COPE. } \\
\text { Tiempo de juego. } \\
\end{array}$ \\
\hline ONDA CERO RADIO & $\begin{array}{l}\text { Herrera en la onda. } \\
\text { Noticias mediodía. } \\
\text { Julia en la onda. } \\
\text { La brújula. } \\
\text { Al primer toque. } \\
\text { Te doy mi palabra. } \\
\text { Noticias fin de semana. }\end{array}$ \\
\hline $\begin{array}{l}\text { RADIO 1. RNE (Radio Nacional de } \\
\text { España) }\end{array}$ & $\begin{array}{l}\text { Las mañanas de RNE. } \\
\text { Diario de las dos. } \\
\text { Esto me suena. } \\
\text { Radiogaceta de los deportes. } \\
24 \text { horas. } \\
\text { No es un día cualquiera. } \\
14 \text { Horas fin de semana. } \\
\text { Tablero deportivo. }\end{array}$ \\
\hline $\begin{array}{l}\text { SER (Sociedad Española de } \\
\text { Radiodifusión) }\end{array}$ & $\begin{array}{l}\text { Hoy por hoy. } \\
\text { Hora } 14 . \\
\text { La ventana. } \\
\text { Hora } 25 . \\
\text { El larguero. } \\
\text { A vivir que son dos días. } \\
\text { Hora } 14 \text { fin de semana. } \\
\text { Punto de fuga. } \\
\text { Carrusel deportivo. }\end{array}$ \\
\hline
\end{tabular}

Tabla 3. Títulos de los programas analizados según los criterios de selección de la población y de la muestra. (Fuente: elaboración propia).

\subsection{El corpus sonoro y las categorías discursivas de orden emergente}

Sobre la muestra de los espacios radiofónicos se ha elaborado un corpus sonoro a partir de una transliteración ortográfica según los enunciados de quienes hablan (Llisterri 2017), que ha sido clasificado en citas, tópicos y categorías según el siguiente protocolo:

1.- CITAS. Selección de los conglomerados en los que se difundan espacios en cuyo contenido haya discursos relacionados con profesiones ejercidas por mujeres. 
2.- TÓPICOS. Detección de las informaciones específicas en las que se realice alguna referencia discursiva sobre las mujeres que trabajan en la carrera judicial.

3.- CATEGORÍAS. Observación del tipo de término utilizado para nombrar a las fiscalas y juezas según una categorización basada en una jerarquización lingüística de género.

El corpus se ha clasificado atendiendo al establecimiento de conceptos teóricos clave que han sido convertidos en categorías conceptuales emergentes estableciéndose de forma inductiva o codificación abierta (Strauss 1987), a partir de la recogida de los datos, del análisis preliminar y de la descripción del contexto en que se han producido (Erlandson et al. 1993), clasificándolas según criterios temáticos en función del asunto de que se trate (Rodríguez, Gil y García, 1996).

\begin{tabular}{|l|l|l|c|}
\hline $\mathbf{C I T A S}$ & TÓPICOS & CATEGORÍAS \\
\hline $\mathbf{1}$ & $\begin{array}{l}\text { La j } 17 \text { de } \\
\text { Diciembre. }\end{array}$ & $\begin{array}{l}\text { La jueza Beatriz Balfagón imputa al } \\
\text { expresidente de la Generalitat de } \\
\text { Catalunya Jordi Pujol de varios } \\
\text { delitos fiscales. }\end{array}$ & jueza \\
\hline $\mathbf{2}$ & $\begin{array}{l}18 \text { de } \\
\text { Diciembre. }\end{array}$ & $\begin{array}{l}\text { Dimisión de Eduardo Torres Dulce } \\
\text { como fiscal general del estado. }\end{array}$ & $\begin{array}{l}\text { candidato/candidata } \\
\text { /candidatura y } \\
\text { fiscal/fiscala/fiscalía. }\end{array}$ \\
\hline $\mathbf{3}$ & $\begin{array}{l}19 \text { de } \\
\text { Diciembre. }\end{array}$ & $\begin{array}{l}\text { Nombramiento de Consuelo Madrigal } \\
\text { como la nueva fiscala general del es- } \\
\text { tado. }\end{array}$ & fiscala \\
\hline
\end{tabular}

Tabla 4. Clasificación de las citas, tópicos y categorías analizadas. (Fuente: elaboración propia).

De acuerdo a esto se establece una categorización lingüística que responde al modelo basado en la codificación de variables cualitativas politómicas de carácter ordinal, es decir, jerarquizándolas según el nivel de sexismo que se exprese a través de los vocablos utilizados para ello según se detalla en la Tabla 5: 


\begin{tabular}{|l|c|c|c|c|}
\cline { 2 - 5 } \multicolumn{2}{l|}{} & $\begin{array}{c}\text { género del } \\
\text { determinante }\end{array}$ & $\begin{array}{c}\text { género del } \\
\text { sustantivo }\end{array}$ & categorías \\
\hline $\begin{array}{l}\text { Discurso } \\
\text { sexista }\end{array}$ & Nivel 1 & masculino & masculino & $\begin{array}{c}\text { El/un/otro juez o } \\
\text { fiscal. }\end{array}$ \\
\cline { 2 - 5 } & Nivel 2 & femenino & masculino & $\begin{array}{c}\text { La/una/otra juez } \\
\text { o fiscal. }\end{array}$ \\
\hline Discurso igualitario & femenino & femenino & $\begin{array}{c}\text { La/una/otra jueza } \\
\text { o fiscala. }\end{array}$ \\
\hline \multicolumn{2}{|l|}{ Discurso inclusivo } & Judicatura o fiscalía \\
\hline
\end{tabular}

Tabla 5. Niveles de sexismo discursivo de representación. (Fuente: elaboración propia).

\section{Resultados}

Posteriormente a la audición del corpus se han observado los siguientes resultados sobre cada una de las siguientes citas:

\section{1.- Cita 1: martes 16 y miércoles 17 de diciembre.}

Tópico: La jueza Beatriz Balfagón imputa al expresidente de la Generalitat de Catalunya Jordi Pujol de varios delitos fiscales.

Categoría: Jueza.

En todos los espacios radiofónicos que hemos estudiado, excepto en uno, hemos escuchado que las referencias a la jueza encargada de este caso han sido construidas utilizando los términos: El juez, la juez o una juez. La excepción la encontramos en este espacio en el que se utilizan los vocablos la jueza y la magistrada aunque estas palabras se pronuncian como parte de un discurso en el que también se utiliza el término el juez de tal forma que podemos escuchar: "El juez cita a varios miembros del clan Pujol, [...] deberán explicarle a la jueza el origen de su fortuna familiar" (ONDA CERO Herrera en la onda, 17 de diciembre).

3.2.- Cita 2: jueves 18 de diciembre.

Tópico: Dimisión de Eduardo Torres Dulce como fiscal general del estado.

Categorías: Candidato/candidata/candidatura y fiscal/fiscala/fiscalía. 
En todos los programas, excepto en los que detallamos posteriormente, se utilizan términos como el candidato, el fiscal, el sustituto o el afiliado, es decir, de género gramatical masculino, para referirse a la persona que potencialmente pueda ocupar el cargo que ha quedado vacante en la fiscalía. En ninguno de ellos se han escuchado términos como candidatura, fiscalia, sustitución o afiliación.

Sin embargo, solamente escuchamos una vez el término fiscala en este programa cuando el presentador anuncia a la audiencia que queda abierto el camino para la elección de: "Fiscal o fiscala", explicando a continuación que: "Se puede decir fiscala, admitido por la RAE, fiscala general del estado [...], el sucesor o sucesora [...]", aunque posteriormente y como parte del mismo discurso señala que: "Se pone en marcha, por tanto, el proceso para elegir sucesor" (RNE, Las mañanas de RNE, 18 de diciembre).

Por su parte, en este espacio escuchamos que: "El sustituto" o "El elegido" para el cargo vacante puede ser: "Hombre o mujer" (SER, Hoy por hoy, 18 de diciembre).

En este otro programa radiofónico se realizan tres entrevistas a otros tantos juristas varones para que opinen sobre esta dimisión desde su óptica profesional. A uno de ellos se le solicita que, aventurándose a las circunstancias, ofrezca a la audiencia el nombre de la persona que a su juicio ocupará el cargo vacante en la fiscalía a lo que contesta argumentando sobre los rumores que circulan en el entorno profesional: "Se habla de dos fiscales de sala: Pilar Valcárcel y Concepción Madrigal" (COPE, La mañana, 18 de diciembre).

Al respecto de esta categoría nos gustaría destacar el siguiente caso. Es un discurso escuchado en el que se comenta la posibilidad de que el nuevo cargo responda a: "Un perfil no tanto técnico-político sino que sea una mujer. Una mujer, en concreto, una fiscal" (RNE, 24 horas, 18 de diciembre).

\section{3.- Cita 3: viernes 19 de diciembre.}

Tópico: Nombramiento de Consuelo Madrigal Martínez-Pereda como la nueva fiscala general del estado.

Categoría: Fiscala. 
En todos los espacios analizados se refieren a Madrigal como fiscal, la fiscal o una fiscal excepto en uno en el que podemos oír que pronuncian la fiscala para referenciarla (SER, Hoy por boy, 19 de Diciembre). Incluso sobre esta información escuchamos que: "El gobierno ya le ha buscado un sustituto, en concreto, una, una mujer" a la fiscalía general del estado (ONDA CERO, Julia en la onda, 19 de Diciembre).

El nombramiento de la nueva fiscala supone una novedad para todos ya que se trata de alguien sin ninguna relevancia mediática y desconocida para la audiencia. Por ello, tras radiar esta noticia los periodistas realizan una semblanza de su persona.

Al citar su biografía, en todos los espacios radiofónicos analizados, se acentúa que se trata de una mujer. Incluso este detalle se realiza posteriormente a la pronunciación de su nombre de pila, Consuelo. Así, escuchamos frases del tipo de: "Consuelo Madrigal, mujer, sustituye a...". Se señala como circunstancia relevante de su nombramiento que al líder del PSOE Pedro Sánchez: "Le gusta que sea una mujer" (RNE, Diario de las dos, 19 de diciembre), añadiendo que ha sido: "Nombrado por el gobierno", y que es: "Muy amable de trato" (RNE, 24 horas, 19 de diciembre).

En estos otros dos espacios se entrevista a la misma fiscala que reflexiona sobre la elección de la nueva fiscala de la misma manera en los dos: "Está muy bien que se haya elegido un fiscal, un fiscal de carrera", añadiendo posteriormente que le parece correcto que haya sido elegida una mujer: "Porque las mujeres están tan preparadas como los hombres, no más preparadas" (SER, Hora 14, 19 de diciembre) y (RNE, 24 horas, 19 de diciembre).

Nos gustaría destacar que en el espacio La ventana de la Cadena SER, concretamente en la sección llamada Unidad de Vigilancia el periodista Isaías Lafuente realiza un ejercicio de supervisión lingüística del castellano recopilando con la ayuda de la audiencia aquellos momentos radiofónicos en los que se ha incurrido en algún error de lenguaje, dicción o locución. Respecto de la noticia que nos ocupa escuchamos en su boca que las palabras: "Fiscala, obispa, papisa, capitana" están recogidas en el diccionario y reclama 
su uso masivo porque aunque existen: "Algunos femeninos que al principio nos resultan extraños, pero después al final, terminamos utilizándolos con una cierta normalidad" (SER, La ventana, 19 de diciembre).

\section{Discusión}

En general, las mujeres que trabajan en la judicatura siguen siendo denominadas utilizando para ello estructuras lingüísticas masculinas. Así, son nombradas con términos como un juez o un fiscal (Discurso sexista de nivel 1) y una juez o una fiscal (Discurso sexista de nivel 2), aunque las instituciones encargadas de velar por un buen uso de nuestro idioma hayan admitido en los diccionarios vocablos como jueza y fiscala que se corresponden con la construcción de un discurso de igualdad de género. Los casos en los que esta última circunstancia acontecen son excepcionales por las escasas ocasiones en las que se producen.

Específicamente en el tópico primero que hemos analizado, es decir, en el que observamos cómo es nombrada una jueza hemos encontrado que todos los discursos son sexistas tanto del nivel 1 como del 2 en cuanto a que lo es bajo las denominaciones el juez o la juez respectivamente. Solamente se ha encontrado un discurso de igualdad en Onda Cero en el que se ha escuchado la jueza aunque haya sido en el mismo contexto en el que se produce uno sexista de nivel 1 ya que en la misma información se pronuncia el juez en referencia a la misma mujer.

En el tópico segundo que hemos analizado hemos encontrado que en todos los espacios se refieren a la persona que potencialmente ocupará el cargo que ha quedado vacante en la fiscalía como sustituto, afiliado, fiscal o candidato que al ser nombres comunes de género gramatical masculino excluyen a una mujer para ello por lo que pertenecen al grado de sexismo 1. Es una forma implícita de construcción de un discurso mediante la que se niega la participación de la mujer al establecer una relación de correspondencia entre un cargo de alto nivel profesional con el universo masculino de manera exclusiva. Si este discurso hubiera sido inclusivo de género debería haberse construido utilizando vocablos como: Sustitución, afiliación, fiscalia o candidatura porque engloban a todos dentro de una estructura discursiva de igualdad simétrica. 
Sin embargo, solamente escuchamos una vez la posibilidad de que este cargo sea ocupado tanto por un varón como por una mujer cuando en Radio Nacional se anuncia a la audiencia que queda abierto el camino para la elección de fiscal o fiscala, aunque le preceda un discurso en el que se pronuncia el término sustituto (Discurso sexista de nivel 1), es decir, que se vuelve a escuchar la masculinización de esta posibilidad porque se debería haber dicho sustitución siendo así un discurso inclusivo de género. Un caso similar es el que acontece en la Cadena SER en la que se cuenta que este cargo puede ser ocupado por un hombre o por una mujer pero ambos englobados bajo el término el sustituto o el elegido (Discurso sexista de nivel 1).

Incluso en la Cadena COPE escuchamos que: "Se habla de dos fiscales de sala: Pilar Valcárcel y Concepción Madrigal", lo que supone también un discurso sexista de nivel 1 porque se debería haber dicho fiscalas ya que son mujeres.

También señalamos que en Radio Nacional se nos cuenta que el nuevo cargo ha de responder a: "Un perfil no tanto técnico-político sino que sea una mujer. Una mujer, en concreto, una fiscal", así comprobamos que la categoría mujer queda excluida de la de persona-con-perfil-técnico-político al establecer en este discurso una disyunción: $\mathrm{O}$ se es mujer o se posee este perfil por lo que lo consideramos de nivel sexista 1. Es una cuestión de exclusión lingüística, una vez más, hacia las mujeres.

En el tercer tópico comprobamos que la nueva fiscala general del estado es nombrada como fiscal (Discurso sexista de nivel 1), la fiscal o una fiscal (Discurso sexista de nivel 2) en todos los audios excepto en la Cadena SER en la que la nombran como la fiscala (Discurso de igualdad). Además, tanto en la Cadena SER como en Radio Nacional, entrevistan a una fiscala que utiliza el término fiscal para referirse al nuevo cargo elegido (Discurso sexista de nivel 1).

Concretamente, en el caso de Onda Cero oímos que: "El gobierno ya le ha buscado un sustituto, en concreto, una, una mujer" a la fiscalía, con lo que se incurre en un grado de sexismo 1 ya que debería haberse utilizado el vocablo sustituta porque ya es conocido que se trata de una mujer. Otro caso de 
idéntico nivel sexista es el que se produce en Radio Nacional al contarnos que ha sido nombrado el nuevo cargo, en vez de nombrada

La excepción la conforma el espacio La Ventana de la Cadena SER en el que se nos cuenta que los términos fiscala y jueza deben utilizarse porque su uso es el correcto aunque resulten extraño por ser novedosos. En este caso se trata de discursos de igualdad.

Por todo lo expuesto podemos afirmar que los espacios radiofónicos difunden una representación discursiva de las mujeres profesionales elaborada desde estructuras masculinizadoras mediante el uso de patrones lingüísticos que son asimétricos en detrimento de lo femenino, es decir, sexistas. Todo esto sucede en un contexto patriarcal y androcéntrico en el que la asignación de roles laborales está basada en el género.

Esta circunstancia tan extendida se produce en los programas y espacios de las cuatro grandes cadenas de radio españolas más importantes sin que hayamos notado una diferencia notable según sea el género o formato de los programas o la titularidad, pública o privada, de las empresas.

\section{Conclusiones}

En esta investigación hemos querido exponer cómo son nombradas en la radio aquellas mujeres que se dedican profesionalmente a la fiscalía y a la judicatura y bajo qué patrones y referentes se construyen los discursos que las referencian. Creemos que estudiar y exponer cómo un medio de comunicación de uso masivo las llama, las clasifica o las bautiza es evaluar el grado de aceptación con el que son reconocidas socialmente.

Así, hemos podido observar que para referenciar discursivamente un universo en el que ellas son mayoría con respecto a los varones ${ }^{3}$ las alocuciones que se construyen y que se difunden a través de las ondas radiofónicas las minusvalora y las coloca en una posición de asimetría y desigualdad con respecto a ellos. Circunstancia que acontece por un uso sexista del lenguaje realizado desde referencias masculinas basadas en estructuras de representación y patrones arcaicos. 
De esta forma, las categorías y las normas de género son recursos utilizados para el fortalecimiento de los estereotipos sobre la feminidad y la masculinidad mediante la construcción y deconstrucción de los significados de género y las formas lingüísticas de las que surgen también las identidades a partir de la performatividad del poder (Greco, 2012), como acontece con el medio radiofónico al descuidar en gran medida al género femenino (Veerkamp 2014).

Sin embargo, teniendo en cuenta que los medios de comunicación quieren explotar su potencial democrático deberían reflejar la diversidad social existente a través de un lenguaje que refleje de la manera más fiel posible las realidades sociales huyendo de su estereotipación y masculinización (UNESCO 2014), ya que estos son injustos y punitivos (Vera y Simelio, 2015).

Así mismo, los profesionales del periodismo deberían conocer las nuevas realidades de género que acontecen para reflejarlas lingüísticamente en los espacios radiofónicos. Todo porque se reconocen a sí mismos como altavoces de la ciudadanía y se identifican con la promoción de la tolerancia y la diversidad cultural (Berganza, Lavín y Piñeiro-Naval 2017).

\section{Referencias bibliográficas}

AIMC, 2015. Asociación para la Investigación de los Medios de Comunicación,. Resumen General Abril 2014 a Marzo 2015 [en línea]. [Consulta 15 Diciembre 2015] Disponible en: https://www.aimc.es/a1 mc-c0nt3nt/uploads/2015/04/resumegm115.pdf

ALAMINOS, A. y CASTEJÓN, J. L., 2006. Elaboración, análisis e interpretación de encuestas, cuestionarios y escalas de opinión. Alicante: Editorial Marfil. ISBN 8426812678.

ALONSO GONZÁLEZ, C.M., 2004. El canto de las sirenas. Comunicación y persuasión en la publicidad radiofónica. Salamanca: Universidad Pontificia de Salamanca. ISBN 9788472995949

AUSTIN, J. L., 1991. Cómo hacer cosas con palabras. $1^{\text {a }}$ ed. Compilado por J. O. Urmson. Trad. por Genaro R. Carrió y Eduardo A. Rabossi. Barcelona: Paidós. ISBN 978-847509-141-9.

BERGANZA, R., LAVÍN, E. y PIÑEIRO-NAVAL, V., 2017. La percepción de los periodistas españoles acerca de sus roles profesionales. Revista Comunicar, 25(51), 83- 
92. [Consulta 2 Febrero 2018]. eISSN 1988-3293. Disponible en: https://doi.org/10.3916/C51-2017-08.

BIERNAT, M. et al., 2018. Effects of dyadic communication on race-based impressions and memory [en línea]. Group Processes \& Intergroup Relations, 21(2), 302-318. [Consulta: 29 Abril 2018]. eISSN 14617188. Disponible en: https://doi.org/10.1177/1368430216663022

BONET, M., 2017. Every Window of Opportunity Needs a Frame to Support It. DAB in Spain: Analog Matters [en línea]. Journal of Radio \& Audio Media, 24(1), 61-76.

[Consulta: $22 \quad$ Febrero 2018]. ISSN

Doi:10.1080/19376529.2017.1297151

BRUNS, A., 2015. Making sense of society through social media [en línea]. Social Media+ Society, 1(1). [Consulta: 8 Febrero 2018]. ISSN 2056-3051. doi: $10.1177 / 2056305115578679$

BURKE, L. E. C. A., 2015. Exploiting the Qualitative Potential of Q Methodology in a Post-Colonial Critical Discourse Analysis [en línea]. International Journal of Qualitative Methods, 14(1), 65-79. [Consulta: 22 Noviembre 2016]. eISSN 16094069. Disponible en: https://doi.org/10.1177/160940691501400107

CASTELLS, M., 2007. Communication, Power and Counter-power in the Network Society [en línea]. International Journal of Communication, 1(1), 238-266. [Consulta: 3 Febrero 2016]. ISSN 1932-8036. Disponible en: http://ijoc.org/index.php/ijoc/article/view/46/35.

CGPJ. CONSEJO GENERAL DEL PODER JUDICIAL, 2015. Informe sobre la estructura de la carrera judicial 2015 [en línea]. [Consulta 5 Marzo 2016]. Disponible en: http:/ / www.ifuturo.org/informe-sobre-la-estructura-de-la-carrera-judicial-2015

ERLANDSON, D. A. et al., 1993. Doing naturalistic inquiry: A guide to methods. California: Sage. ISBN 978-0803949386.

ESTEVE, J.M., 2003. La tercera revolución. La educación en la sociedad del conocimiento. Barcelona: Paidós. ISBN 978-84-493-1472-0.

FASOLI, F., MAZZUREGA, M. y SULPIZIO, S., 2017. When characters impact on dubbing: the role of sexual stereotypes on voice actor/actress' preferences [en línea]. 
Media Psychology, 20(3), 450-476. [Consulta: 19 Enero 2018]. ISSN 1532-785X. Disponible en: https://doi.org/10.1080/15213269.2016.1202840

FAUS, A., 1981. La radio: introducción a un medio desconocido. Madrid: Latina Universitaria. ISBN 847516014X.

FELTHAM-KING, T. y MACLEOD, C., 2016. How content analysis may complement and extend the insights of discourse analysis: an example of research on constructions of abortion in South African newspapers 1978-2005 [en línea]. International journal of qualitative methods, 15(1). [Consulta: 9 Marzo 2017]. ISSN 16094069. doi: 10.1177/1609406915624575

FERNÁNDEZ MARTÍNEZ, D., 2007. From Theory to Method: A methodological approach within critical discourse analysis [en línea]. Critical Discourse Studies, 4(2), 125-140. [Consulta: 6 Mayo 2016]. ISSN 1740-5912. Disponible en: http://dx.doi.org/10.1080/17405900701464790

FERNÁNDEZ SANDE, M. y PEINADO MIGUEL, F., 2012. La empresa radiofónica actual. En: J.I. GALLEGO PÉREZ y M.T. GARCÍA LEIVA coords. Sintonizando el futuro. Radio y producción sonora en el siglo XXI. Madrid: Instituto RTVE, pp.29-60. ISBN 978-84-88788-85-6.

FIRST, A., 2016. Common Sense, Good Sense, and Commercial Television [en línea]. International Journal of Communication, 10(1), 530-548. [Consulta: 19 Junio 2017]. ISSN 1932-8036. Disponible en: http://ijoc.org/index.php/ijoc/article/view/3551/1550.

FLYVBJERG, B., 2006. Five misunderstandings about case-study research. Qualitative inquiry, 12(2), 219-245. [Consulta: 16 Octubre 2016]. ISSN 10778004. Disponible en: https://doi.org/10.1177/1077800405284363

FOUCAULT, M., 2002. El orden del discurso. Barcelona: Tusquets Editores. ISBN 9788483106549.

GALLEGO PÉREZ, J. I., 2010. Podcasting: distribución de contenidos sonoros y nuevas formas de negocio en la empresa radiofónica española. Tesis doctoral. Madrid: Universidad Complutense. [Consulta 21 Abril 2016]. ISBN 978-84-693-6330-0. Disponible en: http://eprints.ucm.es/11205/ 
GARCÍA MESSEGUER, A., 2001. ¿Es sexista la lengua española?. Panace@, 2(3), 20-34. [Consulta: 6 Julio 2016]. ISSN 1537-1964. Disponible en: http://www.medtrad.org/panacea/PanaceaPDFs/Marzo2001.htm

GRECO, L., 2012. Production, circulation and deconstruction of gender norms in LGBTQ speech practices. Discourse Studies, 14(5), 567-585. [Consulta: 11 Noviembre 2014]. e-ISSN 1461-7080. doi: 10.1177/1461445612452229

HENDRIKS VETTEHEN, P. et al., 2017. The Relationship Between Media Content Preferences and Political Participation in 25 European Countries: The Moderating Role of Broadband Penetration and Broadband Access [en línea]. Communication Research. [Consulta: 28 Enero 2018]. e-ISSN 1552-3810 Disponible en: https://doi.org/10.1177/0093650217701033

HERNÁNDEZ SAMPIERI, R., FERNÁNDEZ COLLADO, C. y BAPTISTA LUCIO, P., 2006. Metodología de la investigación. $4^{\mathrm{a}}$ ed. México DF: McGaw Hill. ISBN 970-105753-8.

IBÁÑEZ, T., 2003. El giro lingüístico. En: L. IÑIGUEZ RUEDA ed. Análisis del Discurso: Manual para las ciencias sociales. Barcelona: Editorial UOC, pp. 21-42. ISBN 9788497880008.

KRZYZANOWSKI, M., 2016. Recontextualisation of neoliberalism and the increasingly conceptual nature of discourse: Challenges for critical discourse studies [en línea]. Discourse \& Society, 27(3), 308-321. [Consulta: 2 Abril 2017]. e-ISSN 1460-3624 doi: $10.1177 / 0957926516630901$

LÓPEZ-GUIMERA, G, et. al., 2010. Influence of mass media on body image and eating disordered attitudes and behaviors in females: A review of effects and processes [en línea]. Media Psychology, 13(4), 387-416. [Consulta: 15 Mayo 2015]. ISSN 1532-785X. doi: $10.1080 / 15213269.2010 .525737$.

LOZANO, P., 2006. El tsunami informativo. Panorama comunicativo del siglo XXI. Pamplona: Ediciones Universidad de Navarra. ISBN 978-8431323776.

LLISTERRI, J., 2017. La representación ortográfica de corpus orales [en línea]. Departament de Filologia Espanyola, Barcelona: Universitat Autònoma de Barcelona. Última actualización: 15 Febrero 2018. [Consulta 27 Abril 2018]. Disponible en: http:/ /liceu.uab.cat/ joaquim/language_resources/spoken_res/Repres_ortog_cor p_oral.html 
MARTÍ, J.M., 2004. La programación radiofónica. En: M. P. MARTÍNEZ-COSTA y E. MORENO eds. Programación Radiofónica: arte y técnica del diálogo entre la radio y su audiencia. Barcelona: Ariel, pp.21-45. ISBN 978-8434412996.

MATARESE, M.T. y VAN NIJNAT'TEN, C., 2015. Making a Case for Client Insistence in Social Work Interaction [en línea]. Discourse Processes, 52(8), 670-688. [Consulta: 23 Junio 2017]. e-ISSN1532-6950. doi: 10.1080/0163853X.2014.979974

MOLINER, M., 2007. Diccionario del uso del español. $3^{\text {a }}$ ed. Madrid: Gredos. ISBN 9788424928865

NEWMAN, L. y LEVY, D. A. (eds.), 2014. Reuters Institute digital news report 2014. Tracking the future of news. Reuters Institute. [en línea]. [Consulta 25 Febrero 2016]. ISBN 9781907384127.

Disponible en: http://reutersinstitute.politics.ox.ac.uk/publication/digital-news-report$\underline{2014}$

PATTON, M. Q., 2015. Qualitative Research and Evaluation Methods. 4ª ed. California: Sage Publications. ISBN 978-1-4129-7213-3.

PERONA PÁEZ, J.J., 2012. Las emisoras universitarias en el contexto digital: programación, nuevos medios y hábitos de escucha. En: C. Espino y D. Martín coords. Las radios universitarias, más allá de la radio. Las TIC como recursos de interacción radiofónica. Barcelona: UOC, pp.37-52. ISBN 978-84-9788-989-6.

RAE. REAL ACADEMIA ESPAÑOLA, 2005. Diccionario panbispánico de dudas. [en línea] Madrid: Real Academia Española. [Consulta 22 Mayo 2014]. Disponible en: http://lema.rae.es/dpd/?key=

RAE. REAL ACADEMIA ESPAÑOLA, 2006. Diccionario esencial de la lengua española. Pozuelo de Alarcón (Madrid): Espasa Calpe. ISBN 10:8467023147.

RAE. REAL ACADEMIA ESPAÑOLA, 2010. NGLE, Nueva gramática de la lengua española. Manual. Madrid: Espasa Libros. ISBN 9788467032819.

RAE. REAL ACADEMIA ESPAÑOLA, 2014. DRAE, Diccionario de la lengua española. 23a ed. Madrid: Real Academia Española. ISBN 9788467041897. 
RIBES GUARDIA, F.X., 2001. Las emisoras de radio del estado español en internet: las bitcasters. Tesis doctoral. Barcelona: Universitat Autonòma de Barcelona. [Consulta 12 Mayo 2015]. Disponible en: http://hdl.handle.net/10803/4097. ISBN 8469980866.

RIMAL, R. N., CHUNG, A. H. y DHUNGANA, N., 2015. Media as educator, media as disruptor: Conceptualizing the role of social context in media effects [en línea]. Journal of Communication, 65(5), 863-887. [Consulta: 8 Marzo 2016]. ISSN 0021-9916. Disponible en: https://doi.org/10.1111/jcom.12175.

RODRÍGUEZ, G., GIL, J. y GARCÍA, E., 1996. Métodos de investigación cualitativa. 2ª ed. Málaga: Aljibe. ISBN 978-8487767562.

SANTANDER, D., 2011. Por qué y cómo hacer análisis de discurso [en línea]. Cinta de Moebio. Revista de Epistemología de Ciencias Sociales, (41), 207-224. [Consulta: 28 Septiembre 2015]. ISSN 0717-554X Disponible en: http://www.cintademoebio.uchile.cl/index.php/CDM/article/view/18183/19048

SPERBER, D. y WILSON, D., 2014. La teoría de la relevancia [en línea]. Revista de investigación lingüistica, 7(1), 233-283. [Consulta: 20 Octubre 2016]. e-ISSN 1989-4554 Disponible en: http://revistas.um.es/ril/article/view/6691/6491

STRAUSS, A., 1987. Qualitative Analysis for Social Scientists. Cambridge: University Press. ISBN 978-0521338066.

TANNEN, D., 1996. Género y discurso. Barcelona: Paidós. ISBN 84-493-0292-7

TAYLOR, S.J. y BODGAN, R., 1987. Introducción a los métodos cualitativos de investigación. $2^{\mathrm{a}}$ ed. Barcelona: Paidós. ISBN 978-84-7509-816-6.

TENORIO, I., 2012. La nueva radio. Manual completo del radiofonista 2.0. $2^{\mathrm{a}}$ ed. Barcelona: Marcombo. ISBN 978-8426717771.

UNESCO, 2014. Indicadores de UNESCO para medios de comunicación. Marco de indicadores para evaluar la sensibilidad en materia de género en las operaciones y contenidos mediáticos [en línea]. París: UNESCO. [Consulta 22 Abril 2016]. ISBN 978-92-3-300007-0. Disponible en:

http://unesdoc.unesco.org/images/0023/002310/231069s.pdf 
VALLES, M., 2000. Técnicas cualitativas de investigación social. Reflexión metodológica y práctica profesional. Madrid: Síntesis. ISBN 84-7738-449-5.

VAN DIJK, T., 1990. La noticia como discurso. Barcelona: Paidós. ISBN 9788475096223.

VAN DIJK, T., 2003. La multidisciplinariedad del análisis crítico del discurso: un alegato en favor de la diversidad. En: R. WODAK y M. MEYER, comps. Métodos de análisis crítico del discurso. Madrid: Gedisa, pp.143-178. ISBN 978-8474329704.

VEERKAMP, H., 2014. Feminist Frequencies: Why Radio Needs Feminism. Journal of Radio \& Audio Media, 21(2), 307-315. [Consulta: 17 Enero 2016]. ISSN 1937-6529. doi:10.1080/19376529.2014.950154.

VERA, T. y SIMELIO, N., 2015. ¿Quién figura en las noticias? Proyecto de Monitoreo Global de Medios 2015. Informe Nacional [en línea]. [Consulta 23 de Mayo 2017]. Disponible en: http://hdl.handle.net/10630/10773

VIVANCO, M., 2005. Muestreo estadístico. Diseño y aplicaciones. Santiago de Chile: Editorial Universitaria. ISBN 956-11-1803-3.

VIVANCO, M., 2006. Diseños de muestras en investigación social. En: M. CANALES CERÓN coord. y ed. Metodologías de la investigación social. Introducción a los oficios. Santiago: LOM, pp.141-168. ISBN 956-282-840-9.

VOSS, J.F. y VAN DYKE, J.A., 2001. Argumentation in Psychology: Background Comments [en línea]. Discourse Processes, 32(2-3), 89-111. [Consulta: 28 Junio 2015]. e-ISSN 1532-6950. doi: 10.1080/0163853X.2001.9651593

WITTIG, M., 2005. El pensamiento heterosexual y otros ensayos. $2^{\mathrm{a}}$ ed. Madrid: Egales. ISBN 978-8495346971.

WODAK, R., 2006. Mediation between discourse and society: assessing cognitive approaches in CDA [en línea]. Discourse Studies, 8(1), 179-190. [Consulta: 7 Octubre 2017]. e-ISSN 1461-7080.

doi: $10.1177 / 1461445606059566$

\section{Notas}


NOTA ${ }^{1}$. En los informes de la agencia Reuters sobre los medios con mayor consumo semanal en España en 2014 figuran entre los 13 primeros tanto los servicios informativos de la SER como las noticias de Onda Cero. Con respecto a 2015 son las cadenas SER, Onda Cero, RNE y COPE, en este orden, las que tienen un mayor consumo en informaciones digitales (Newman et al., 2014).

NOTA $^{2}$. El grado de penetración de la radio en España en el período desde abril de 2014 hasta marzo de 2015 es del 60,4\% en comparación con otros medios. La audiencia de las cadenas generalistas fue: SER 4.444.000, Onda Cero 2.394.000, COPE 1.811.000 y RNE 1.239.000, compuesta por un $48 \%$ de mujeres y un $52 \%$ de varones. Entre las cuatro suman el $77 \%$ de la audiencia de las radios españolas de programación convencional o generalista con un consumo de 48 minutos por persona y día (AIMC, 2015).

NOTA ${ }^{3}$. El informe realizado por el Consejo General del Poder Judicial sobre la estructura de la carrera judicial en España señala que a 1 de enero de 2015 un 52\% del personal de su plantilla está compuesta por mujeres (CGPJ, 2015). 